KYUNGPOOK Math. J. 54(2014), 43-63

http://dx.doi.org/10.5666/KMJ.2014.54.1.43

\title{
Fourier Cosine and Sine Transformable Boehmians
}

Chinnaraman Ganesan

Department of Mathematics, V. H. N. S. N. College, Virudhunagar-626 001, India e-mail : c.ganesan28@yahoo.com

RAJAKUmar RoOPKUMAR*

Department of Mathematics, Alagappa University, Karaikudi- 630 004, India

e-mail : roopkumarr@rediffmail.com

ABstract. The range spaces of Fourier cosine and sine transforms on $L^{1}([0, \infty))$ are characterized. Using Fourier cosine and sine type convolutions, Fourier cosine and sine transformable Boehmian spaces have been constructed, which properly contain $L^{1}([0, \infty)$ ). The Fourier cosine and sine transforms are extended to these Boehmian spaces consistently and their properties are established.

\section{Introduction}

Let $\mathbb{N}, \mathbb{R}$ and $\mathbb{C}$ be the sets of natural, real and complex numbers respectively. Let $C([0, \infty))$ be the Fréchet space of all continuous complex valued functions on $[0, \infty)$ with the sequence of semi-norms $\sup _{x \in[0, n]}|f(x)|, \forall f \in C([0, \infty)), n \in \mathbb{N}$. Let $\left(C_{0}([0, \infty)),\|\cdot\|_{\infty}\right)$ be the normed linear space of all continuous complex valued functions on $[0, \infty)$ vanishing at infinity, where $\|\cdot\|_{\infty}$ is the supremum norm. Let $L^{1}([0, \infty))$ be the normed linear space of all Lebesgue measurable functions on $[0, \infty)$ with $\|f\|_{1}=\int_{0}^{\infty}|f(s)| d s<\infty$.

For a suitable function $f$ on $[0, \infty)$, the Fourier cosine and sine transforms [11] are defined by

$$
\mathcal{C}(f)(t)=\int_{0}^{\infty} f(s) \cos (s t) d s \text { and } \mathcal{S}(f)(t)=\int_{0}^{\infty} f(s) \sin (s t) d s, \quad \forall t \in[0, \infty),
$$

respectively. For more details about Fourier cosine and sine transforms, we refer the reader to $[11,12]$ and for various types of convolutions and convolution theorems for Fourier cosine and sine transforms we refer to $[2,4]$. On the other hand, motivated

* Corresponding Author.

Received May 16, 2011; revised March 16, 2013; accepted November 13, 2013.

2000 Mathematics Subject Classification: 44A15, 44A35.

Key words and phrases: Fourier cosine and sine transforms, convolution, Boehmians. 
by the concept of regular operators [1], the theory of Boehmians was introduced in [5]. Two notions of convergence on the space of Boehmians and their properties are discussed in [6]. Further, many integral transforms have been defined and studied on various Boehmian spaces. A complete list of references on Boehmians is available in http://math.ucf.edu/ piotr/Boehmians.pdf.

In the present article, we construct a Fourier cosine transformable Boehmian space and a Fourier sine transformable Boehmian space by proving all the required auxiliary results. Further, we extend the Fourier cosine and sine transform on these Boehmian spaces consistently and discuss their properties.

This paper is organized as follows. In Section 2, by recalling the theory of Fourier cosine and sine transforms from [11], we first point out that Fourier cosine and sine transforms are linear, continuous, injective mappings from $L^{1}([0, \infty))$ into $C_{0}([0, \infty))$. Then, we characterize the range of Fourier cosine and sine transforms on $L^{1}([0, \infty))$ and we also prove some results involving Fourier cosine and sine convolutions. In Section 3, we construct Fourier cosine transformable and Fourier sine transformable Boehmian spaces $\mathcal{B}_{c}^{1}$ and $\mathcal{B}_{s}^{1}$, by proving the required auxiliary results. In Section 4, we extend the Fourier cosine and sine transforms as linear one-to-one continuous mappings, respectively, from $\mathcal{B}_{c}^{1}$ and $\mathcal{B}_{s}^{1}$ into $C([0, \infty))$. We also obtain characterization theorems for the range of extended Fourier cosine and sine transforms on Boehmians.

\section{Fourier Cosine and Sine Transforms on $L^{1}([0, \infty))$}

We first observe that $\mathcal{C}(f) \in C_{0}([0, \infty))$ and $\|\mathcal{C}(f)\|_{\infty} \leq\|f\|_{1}, \forall f \in L^{1}([0, \infty))$. Indeed, for $f \in L^{1}([0, \infty))$, consider $g: \mathbb{R} \rightarrow \mathbb{C}$ defined by $g(x)=f(x), \forall x \geq 0$ and $g(x)=0, \forall x<0$. Then $g \in L^{1}(\mathbb{R})$ and hence its Fourier transform $\mathcal{F}(g) \in C_{0}(\mathbb{R})$. By using the definition, we get

$$
\begin{aligned}
\mathcal{C}(f)(t) & =\int_{0}^{\infty} f(s) \cos (s t) d s=\int_{0}^{\infty} f(s)\left(\frac{e^{i s t}+e^{-i s t}}{2}\right) d s \\
& =\frac{1}{2}\left(\int_{0}^{\infty} f(s) e^{i s t} d s+\int_{0}^{\infty} f(s) e^{-i s t} d s\right)=\frac{\mathcal{F}(g)(-t)+\mathcal{F}(g)(t)}{2}
\end{aligned}
$$

which clearly shows that $\mathcal{C}(f) \in C_{0}([0, \infty))$. From the definition of cosine transform, we also get $|\mathcal{C}(f)(t)| \leq \int_{0}^{\infty}|f(s)||\cos (s t)| d s \leq\|f\|_{1}, \forall t \in[0, \infty)$, which in turn implies that $\|\mathrm{C}(f)\|_{\infty} \leq\|f\|_{1}$. Further, from the identity $f(s)=\int_{0}^{\infty} \mathcal{C}(f)(t) \cos (t s) d t, \quad \forall s \in$ $[0, \infty)[11]$, it follows that $\mathcal{C}(\mathcal{C}(f))=f$, whenever $f, \mathcal{C}(f) \in L^{1}([0, \infty))$. From these observations, we have the following proposition.

Proposition 2.1. The Fourier cosine transform $\mathcal{C}: L^{1}([0, \infty)) \rightarrow C_{0}([0, \infty))$ is linear, one-to-one and continuous.

Similarly, we also have the following proposition.

Proposition 2.2. The Fourier sine transform $\mathcal{S}: L^{1}([0, \infty)) \rightarrow C_{0}([0, \infty))$ is 
linear, one-to-one and continuous.

Definition 2.3.([11]) The Fourier cosine type convolution is defined for $f, g \in$ $L^{1}([0, \infty))$ by $\left(f *_{c} g\right)(x)=\frac{1}{2} \int_{0}^{\infty}[f(x+y)+f(|x-y|)] g(y) d y, \forall x \geq 0$.

Lemma 2.4. If $f, g \in L^{1}([0, \infty))$, then $f *_{c} g \in L^{1}([0, \infty))$.

Proof. By using Fubini's theorem, we obtain

$$
\begin{aligned}
& \left\|f *_{c} g\right\|_{1} \leq \frac{1}{2} \int_{0}^{\infty}\left(\int_{0}^{\infty}|f(x+y)||g(y)| d y+\int_{0}^{\infty}|f(|x-y|)||g(y)| d y\right) d x \\
= & \frac{1}{2}\left(\int_{0}^{\infty} \int_{0}^{\infty}|f(x+y)||g(y)| d x d y+\int_{0}^{\infty} \int_{0}^{\infty}|f(|x-y|)||g(y)| d x d y\right) \\
= & \frac{1}{2}\left(\int_{0}^{\infty} \int_{y}^{\infty}|f(z)||g(y)| d z d y+\int_{0}^{\infty} \int_{-y}^{\infty}|f(|z|)||g(y)| d z d y\right) \\
= & \frac{1}{2}\left(\int_{0}^{\infty} \int_{y}^{\infty}|f(z)||g(y)| d z d y+\int_{0}^{\infty} \int_{-y}^{0}|f(-z)||g(y)| d z d y+\int_{0}^{\infty} \int_{0}^{\infty}|f(z)||g(y)| d z d y\right) \\
= & \frac{1}{2}\left(\int_{0}^{\infty} \int_{y}^{\infty}|f(z)||g(y)| d z d y+\int_{0}^{\infty} \int_{0}^{y}|f(z)||g(y)| d z d y+\int_{0}^{\infty} \int_{0}^{\infty}|f(z)||g(y)| d z d y\right) \\
= & \int_{0}^{\infty} \int_{0}^{\infty}|f(z)||g(y)| d z d y \\
= & \|f\|_{1}\|g\|_{1}, \text { and hence } f *_{c} g \in L^{1}([0, \infty)) .
\end{aligned}
$$

Lemma 2.5. If $f_{n} \rightarrow f$ as $n \rightarrow \infty$ in $L^{1}([0, \infty))$ and if $g \in L^{1}([0, \infty))$, then $f_{n} *_{c} g \rightarrow f *_{c} g$ as $n \rightarrow \infty$ in $L^{1}([0, \infty))$.

Proof. From the proof of Lemma 2.4, we have the estimate

$$
\left\|\left(f_{n}-f\right) *_{c} g\right\|_{1} \leq\left\|f_{n}-f\right\|_{1}\|g\|_{1} .
$$

Since $f_{n} \rightarrow f$ as $n \rightarrow \infty$ in $L^{1}([0, \infty))$, the right hand side of (2.1) tends to zero as $n \rightarrow \infty$. Hence the lemma follows.

Lemma 2.6. If $f, g \in L^{1}([0, \infty))$, then $f *_{c} g=g *_{c} f$.

Proof. Let $t \in[0, \infty)$ be arbitrary. 


$$
\begin{aligned}
\left(f *_{c} g\right)(t) & =\int_{0}^{\infty} f(x+t) g(x) d x+\int_{0}^{\infty} f(|x-t|) g(x) d x \\
& =\int_{t}^{\infty} f(y) g(y-t) d y+\int_{-t}^{\infty} f(|z|) g(z+t) d z \\
& =\int_{t}^{\infty} f(y) g(y-t) d y+\int_{-t}^{0} f(-z) g(z+t) d z+\int_{0}^{\infty} f(z) g(z+t) d z \\
& (\text { by putting } y=-z \text { in the second integral) } \\
= & \int_{t}^{\infty} f(y) g(y-t) d y+\int_{0}^{t} f(y) g(t-y) d y+\int_{0}^{\infty} f(z) g(z+t) d z \\
= & \int_{0}^{\infty} f(y) g(|y-t|) d y+\int_{0}^{\infty} f(z) g(z+t) d z \\
& =\int_{0}^{\infty} f(y)[g(|y-t|)+g(y+t)] d y \\
& =\left(g *_{c} f\right)(t) .
\end{aligned}
$$

Lemma 2.7. If $f, \varphi, \psi \in L^{1}([0, \infty))$, then $\left(f *_{c} \varphi\right) *_{c} \psi=f *_{c}\left(\varphi *_{c} \psi\right)$.

Proof. By using Fubini's theorem and suitable change of variables, we get $4\left[f *_{c}\left(\varphi *_{c} \psi\right)\right](t)$

$=2 \int_{0}^{\infty}[f(t+x)+f(|t-x|)]\left(\varphi *_{c} \psi\right)(x) d x$

$=\int_{0}^{\infty} f(t+x) \int_{0}^{\infty}[\varphi(x+y)+\varphi(|x-y|)] \psi(y) d y d x$

$+\int_{0}^{\infty} f(|t-x|) \int_{0}^{\infty}[\varphi(x+y)+\varphi(|x-y|)] \psi(y) d y d x$

$=\int_{0}^{\infty} \psi(y) \int_{0}^{\infty} f(t+x) \varphi(x+y) d x d y+\int_{0}^{\infty} \psi(y) \int_{0}^{\infty} f(|t-x|) \varphi(x+y) d x d y$

$+\int_{0}^{\infty} \psi(y) \int_{0}^{\infty} f(t+x) \varphi(|x-y|) d x d y+\int_{0}^{\infty} \psi(y) \int_{0}^{\infty} f(|t-x|) \varphi(|x-y|) d x d y$

$=\int_{0}^{\infty} \psi(y) \int_{y}^{\infty} f(t+z-y) \varphi(z) d z d y+\int_{0}^{\infty} \psi(y) \int_{y}^{\infty} f(|t+y-z|) \varphi(z) d z d y$

$+\int_{0}^{\infty} \psi(y) \int_{-y}^{\infty} f(t+z+y) \varphi(|z|) d z d y+\int_{0}^{\infty} \psi(y) \int_{-y}^{\infty} f(|t-z-y|) \varphi(|z|) d z d y$

$=\int_{0}^{\infty} \psi(y) \int_{y}^{\infty} f(t+z-y) \varphi(z) d z d y+\int_{0}^{\infty} \psi(y) \int_{y}^{\infty} f(|t+y-z|) \varphi(z) d z d y$

$+\int_{0}^{\infty} \psi(y)\left(\int_{-y}^{0} f(t+z+y) \varphi(-z) d z+\int_{0}^{\infty} f(t+z+y) \varphi(z) d z\right) d y$

$+\int_{0}^{\infty} \psi(y)\left(\int_{-y}^{0} f(|t-z-y|) \varphi(-z) d z+\int_{0}^{\infty} f(|t-z-y|) \varphi(z) d z\right) d y$

$=\int_{0}^{\infty} \psi(y) \int_{y}^{\infty} f(t+z-y) \varphi(z) d z d y+\int_{0}^{\infty} \psi(y) \int_{y}^{\infty} f(|t+y-z|) \varphi(z) d z d y$ 


$$
\begin{aligned}
& +\int_{0}^{\infty} \psi(y)\left(\int_{0}^{y} f(t-z+y) \varphi(z) d z+\int_{0}^{\infty} f(t+z+y) \varphi(z) d z\right) d y \\
& +\int_{0}^{\infty} \psi(y)\left(\int_{0}^{y} f(|t+z-y|) \varphi(z) d z+\int_{0}^{\infty} f(|t-z-y|) \varphi(z) d z\right) d y \\
& =\int_{0}^{\infty} \psi(y) \int_{0}^{\infty} f(t+z+y) \varphi(z) d z d y+\int_{0}^{\infty} \psi(y) \int_{0}^{\infty} f(|t-z-y|) \varphi(z) d z d y \\
& +\int_{0}^{\infty} \psi(y)\left(\int_{y}^{\infty} f(t+z-y) \varphi(z) d z+\int_{0}^{y} f(|t+z-y|) \varphi(z) d z\right) d y \\
& +\int_{0}^{\infty} \psi(y)\left(\int_{y}^{\infty} f(|t+y-z|) \varphi(z) d z+\int_{0}^{y} f(t-z+y) \varphi(z) d z\right) d y \\
& =\int_{0}^{\infty} \psi(y)\left(\int_{0}^{\infty} f(t+z+y) \varphi(z) d z\right) d y+\int_{0}^{\infty} \psi(y)\left(\int_{0}^{\infty} f(|t-z-y|) \varphi(z) d z\right) d y \\
& +\int_{0}^{\infty} \psi(y)\left(\int_{0}^{\infty} f(|t+z-y|) \varphi(z) d z\right) d y+\int_{0}^{\infty} \psi(y)\left(\int_{0}^{\infty} f(|t+y-z|) \varphi(z) d z\right) d y \\
& =\int_{0}^{\infty} \psi(y)\left(\int_{0}^{\infty} f(t+z+y) \varphi(z) d z\right) d y+\int_{0}^{\infty} \psi(y)\left(\int_{0}^{\infty} f(|t+y-z|) \varphi(z) d z\right) d y \\
& +\int_{0}^{t} \psi(y) \int_{0}^{\infty} f(|t-y+z|) \phi(z) d z d y+\int_{t}^{\infty} \psi(y) \int_{0}^{\infty} f(|t-y+z|) \phi(z) d z d y \\
& +\int_{0}^{t} \psi(y) \int_{0}^{\infty} f(|t-y-z|) \phi(z) d z d y+\int_{t}^{\infty} \psi(y) \int_{0}^{\infty} f(|t-y-z|) \phi(z) d z d y \\
& =\int_{0}^{\infty} \psi(y) \int_{0}^{\infty}[f(t+y+z)+f(|t+y-z|)] \varphi(z) d z d y \\
& +\int_{0}^{t} \psi(y) \int_{0}^{\infty} f(t-y+z) \phi(z) d z d y+\int_{t}^{\infty} \psi(y) \int_{0}^{\infty} f(|t-y+z|) \phi(z) d z d y \\
& +\int_{0}^{t} \psi(y) \int_{0}^{\infty} f(|t-y-z|) \phi(z) d z d y+\int_{t}^{\infty} \psi(y) \int_{0}^{\infty} f(y-t+z) \phi(z) d z d y \\
& =2 \int_{0}^{\infty} \psi(y)\left(f *_{c} \phi\right)(t+y) d y \\
& +\int_{0}^{t} \psi(y) \int_{0}^{\infty} f(t-y+z) \phi(z) d z d y+\int_{t}^{\infty} \psi(y) \int_{0}^{\infty} f(y-t+z) \phi(z) d z d y \\
& +\int_{0}^{t} \psi(y) \int_{0}^{\infty} f(|t-y-z|) \phi(z) d z d y+\int_{t}^{\infty} \psi(y) \int_{0}^{\infty} f(|t-y+z|) \phi(z) d z d y \\
& =2 \int_{0}^{\infty} \psi(y)\left(f *_{c} \phi\right)(t+y) d y \\
& +\int_{0}^{\infty} \psi(y) \int_{0}^{\infty} f(|t-y|+z) \phi(z) d z d y+\int_{0}^{\infty} \psi(y) \int_{0}^{\infty} f(|| t-y|-z|) \phi(z) d z d y \\
& =2 \int_{0}^{\infty} \psi(y)\left(f *_{c} \phi\right)(t+y) d y+2 \int_{0}^{\infty} \psi(y)\left(f *_{c} \phi\right)(|t-y|) d y \\
& =2 \int_{0}^{\infty} \psi(y)\left[\left(f *_{c} \phi\right)(t+y)+\left(f *_{c} \phi\right)(|t-y|)\right] d y \\
& =4\left[\left(f *_{c} \phi\right) *_{c} \psi\right](t) \text {. }
\end{aligned}
$$


Theorem 2.8. (Convolution theorem for Fourier cosine transform [11]) If $f, g \in$ $L^{1}([0, \infty))$, then $\mathrm{C}\left(f *_{c} g\right)=\mathcal{C}(f) \cdot \mathcal{C}(g)$.

Lemma 2.9. If $f \in L^{1}([0, \infty))$, then $\int_{0}^{\infty}|f(x+y)-f(x)| d x \rightarrow 0$ as $y \rightarrow 0$ and $\int_{0}^{\infty}|f(|x-y|)-f(x)| d x \rightarrow 0$ as $y \rightarrow 0$.

Proof. Let $\epsilon>0$ be arbitrary. For $f \in L^{1}([0, \infty))$ and $t \in[0, \infty)$, let

$$
f_{t}(s)= \begin{cases}0 & 0 \leq s<t \\ f(s-t), & s \geq t\end{cases}
$$

By using the change of variable, $u=x+y$, we get

$$
\int_{0}^{\infty}|f(x+y)-f(x)| d s=\int_{y}^{\infty}|f(u)-f(u-y)| d u \leq \int_{0}^{\infty}|f(u)-f(u-y)| d u=\left\|f_{y}-f\right\|_{1} .
$$

Since the space $C_{c}([0, \infty))$ of all continuous functions on $[0, \infty)$ with compact supports is dense in $L^{1}([0, \infty))$, for the given $\epsilon>0$, we find $g \in C_{c}([0, \infty))$ such that $\|f-g\|_{1}<\frac{\epsilon}{3}$. Choose a compact subset $H$ of $[0, \infty)$ which contains the supports of $g$ and $g_{y}, \forall y \in[0,1)$. Since $g$ is uniformly continuous on $[0, \infty)$, there exists $0<\delta<1$ such that $|s-t|<\delta$ implies that $|g(s)-g(t)|<\frac{\epsilon}{3 M}$, where $M<\infty$ is greater than the Lebesgue measure of $H$. Now for $0 \leq y<\delta$, we have

$$
\begin{aligned}
\left\|f_{y}-f\right\|_{1} & \leq\left\|f_{y}-g_{y}\right\|_{1}+\left\|g_{y}-g\right\|_{1}+\|g-f\|_{1} \\
& \leq 2 \frac{\epsilon}{3}+\left\|g_{y}-g\right\|_{1} \quad\left(\text { since }\left\|f_{y}-g_{y}\right\|_{1}=\|g-f\|_{1}<\frac{\epsilon}{3}\right) \\
& \leq 2 \frac{\epsilon}{3}+\int_{0}^{\infty}|g(u)-g(u-y)| d u \\
& \leq 2 \frac{\epsilon}{3}+\int_{H} \frac{\epsilon}{3 M} d u<\epsilon .
\end{aligned}
$$

Therefore, $\int_{0}^{\infty}|f(x+y)-f(x)| d x \rightarrow 0$ as $y \rightarrow 0$. Next we observe that

$$
\int_{0}^{\infty}|f(|x-y|)-f(x)| d x=\int_{0}^{y}|f(y-x)-f(x)| d x+\int_{y}^{\infty}|f(x-y)-f(x)| d x .
$$

Using the previous argument, the second term in (2.2) tends to 0 as $y \rightarrow 0$. On the other hand, for $0 \leq x \leq y<\frac{\delta}{3}$, we have $|y-x-x| \leq 3 y<\delta$ and hence $|g(y-x)-g(x)|<\frac{\epsilon}{3 M}$. Therefore, 


$$
\begin{aligned}
& \int_{0}^{y}|f(y-x)-f(x)| d x \\
& =\int_{0}^{y}|f(y-x)-g(y-x)+g(y-x)-g(x)+g(x)-f(x)| d x \\
& \leq 2\|f-g\|_{1}+\int_{0}^{y}|g(y-x)-g(x)| d x \\
& <2 \frac{\epsilon}{3}+\int_{H} \frac{\epsilon}{3 M} d x<\epsilon .
\end{aligned}
$$

Thus the lemma follows.

Theorem 2.10. Let $f \in L^{1}([0, \infty))$ and let $\left(g_{n}\right)$ be a sequence of functions defined on $[0, \infty)$ such that

$(P 1) \int_{0}^{\infty} g_{n}(y) d y=1, \forall n \in \mathbb{N},(P 2) \int_{0}^{\infty}\left|g_{n}(y)\right| d y \leq M$ for some $M>0, \forall n \in \mathbb{N}$, and (P3) $\lim _{n \rightarrow \infty} \int_{\delta}^{\infty}\left|g_{n}(y)\right| d y=0$, for every $\delta>0$. Then, $f *_{c} g_{n} \rightarrow f$ as $n \rightarrow \infty$ in $L^{1}([0, \infty))$.

Proof. Let $\epsilon>0$ be given. Using Lemma 2.9, we choose a $\delta>0$ such that

$$
\int_{0}^{\infty}|f(x+y)-f(x)| d x<\frac{\epsilon}{2 M} \text { and } \int_{0}^{\infty}|f(|x-y|)-f(x)| d x<\frac{\epsilon}{2 M}, \forall y \in[0, \delta) .
$$

Next, using the property $(P 3)$ of $\left(g_{n}\right)$, we also find a positive integer $N$ such that $\int_{\delta}^{\infty}\left|g_{n}(y)\right| d y<\frac{\epsilon}{4\left(\|f\|_{1}+1\right)}, \forall n \geq N$. Applying Fubini's theorem, for $0 \leq y<\delta$ and $n \geq N$, we obtain that

$$
\begin{aligned}
& \left\|f *_{c} g_{n}-f\right\|_{1} \\
& =\int_{0}^{\infty}\left|\frac{1}{2} \int_{0}^{\infty}[f(x+y)+f(|x-y|)] d y-f(x) \int_{0}^{\infty} g_{n}(y) d y\right| d x, \quad(\operatorname{using}(P 1)) \\
& =\frac{1}{2} \int_{0}^{\infty}\left|\int_{0}^{\infty}[f(x+y)-f(x)+f(|x-y|)-f(x)] g_{n}(y) d y\right| d x \\
& \leq \frac{1}{2} \int_{0}^{\infty}\left(\int_{0}^{\infty}|f(x+y)-f(x)|\left|g_{n}(y)\right| d y+\int_{0}^{\infty}|f(|x-y|)-f(x)|\left|g_{n}(y)\right| d y\right) d x \\
& \leq \frac{1}{2}\left(\int_{0}^{\infty} \int_{0}^{\infty}|f(x+y)-f(x)| d x\left|g_{n}(y)\right| d y+\int_{0}^{\infty} \int_{0}^{\infty}|f(|x-y|)-f(x)| d x\left|g_{n}(y)\right| d y\right) \\
& <\frac{1}{2}\left(2 \frac{\epsilon}{2 M} \int_{0}^{\delta}\left|g_{n}(y)\right| d y+\int_{\delta}^{\infty}\left|g_{n}(y)\right|\left[\int_{0}^{\infty}|f(x+y)-f(x)| d x\right] d y\right. \\
& \left.+\int_{\delta}^{\infty}\left|g_{n}(y)\right|\left[\int_{0}^{\infty}|f(|x-y|)-f(x)| d x\right] d y\right) \\
& \leq \frac{\epsilon}{2 M} \int_{0}^{\delta}\left|g_{n}(y)\right| d y+\frac{1}{2} \int_{\delta}^{\infty}\left|g_{n}(y)\right|\left(\int_{0}^{\infty}|f(x+y)| d x+2 \int_{0}^{\infty}|f(x)| d x+\int_{0}^{\infty}|f(|x-y|)| d x\right)
\end{aligned}
$$


$\leq \frac{\epsilon}{2 M} \int_{0}^{\delta}\left|g_{n}(y)\right| d y+\frac{1}{2} \int_{\delta}^{\infty}\left|g_{n}(y)\right|\left(\int_{y}^{\infty}|f(z)| d z+2 \int_{0}^{\infty}|f(x)| d x+\int_{-y}^{\infty}|f(|z|)| d z\right)$

$\leq \frac{\epsilon}{2 M} \int_{0}^{\delta}\left|g_{n}(y)\right| d y+\frac{1}{2} \int_{\delta}^{\infty}\left|g_{n}(y)\right|\left(\int_{y}^{\infty}|f(z)| d z+2\|f\|_{1}+\int_{0}^{y}|f(z)| d z+\|f\|_{1}\right)$

$\leq \frac{\epsilon}{2 M} \int_{0}^{\infty}\left|g_{n}(y)\right| d y+\frac{1}{2} \int_{\delta}^{\infty}\left|g_{n}(y)\right| 4\|f\|_{1} d y$

$=\frac{\epsilon}{2}+2\|f\|_{1} \int_{\delta}^{\infty}\left|g_{n}(y)\right| d y, \quad($ using $(P 2))$

$<\frac{\epsilon}{2}+\frac{\epsilon}{2\left(\|f\|_{1}+1\right)}\|f\|_{1}<\epsilon$.

Thus the theorem follows.

Theorem 2.11. A necessary and sufficient condition for $F \in C_{0}([0, \infty))$ to be in the range of the Fourier cosine transform on $L^{1}([0, \infty))$ is that $\left(f_{n}\right)$ converges in $L^{1}([0, \infty))$, where

$$
f_{n}(x)=\int_{0}^{n}\left(1-\frac{t}{n}\right) F(t) \cos (x t) d t, \forall x \in[0, \infty) \text { and } n=1,2,3, \cdots
$$

Proof. Assume that the sequence $\left(f_{n}\right)$ of functions as defined in (2.3) converges to $f$ in $L^{1}([0, \infty))$. We first observe that if $k_{n}(t)=\chi_{[0, n]}(t)\left(1-\frac{t}{n}\right)$ and $h_{n}(t)=$ $k_{n}(t) \cdot F(t), \forall t \in[0, \infty), n \in \mathbb{N}$, then we have

- $h_{n} \in L^{1}([0, \infty)), \forall n \in \mathbb{N}$,

- $\mathcal{C}\left(h_{n}\right)=f_{n} \in L^{1}([0, \infty)), \forall n \in \mathbb{N}$, and hence $\mathcal{C}\left(f_{n}\right)=h_{n}, \forall n \in \mathbb{N}$,

- $\mathcal{C}\left(k_{n}\right)(u)=\int_{0}^{n}\left(1-\frac{t}{n}\right) \cos (u t) d t=\frac{1-\cos (n u)}{n u^{2}}=\pi \frac{n}{2 \pi}\left(\frac{\sin \left(\frac{n x}{2}\right)}{\frac{n x}{2}}\right)^{2}=\pi g_{n}(u)$, where $g_{n}(x)=\frac{n}{2 \pi}\left(\frac{\sin \left(\frac{n x}{2}\right)}{\frac{n x}{2}}\right)^{2}, \forall x \geq 0$.

Since $\mathcal{C}: L^{1}([0, \infty)) \rightarrow C_{0}([0, \infty))$ is continuous, we have $\mathcal{C}(f)=\lim _{n \rightarrow \infty} \mathcal{C}\left(f_{n}\right)=$ $\lim _{n \rightarrow \infty} h_{n}=F$. Conversely, assume that there exists $f \in L^{1}([0, \infty))$ such that $F=$ $\stackrel{n \rightarrow \infty}{\mathcal{C}(f) \text {. Using Fubini's theorem, we get }}$ 


$$
\begin{aligned}
f_{n}(x) & =\int_{0}^{n}\left(1-\frac{t}{n}\right) F(t) \cos (x t) d t \\
& =\int_{0}^{n}\left(1-\frac{t}{n}\right)\left(\int_{0}^{\infty} f(y) \cos (y t) d y\right) \cos (x t) d t, \\
& =\frac{1}{2} \int_{0}^{\infty} f(y)\left(\int_{0}^{n}\left(1-\frac{t}{n}\right)[\cos (x+y) t+\cos (|x-y| t)] d t\right) d y \\
& =\frac{1}{2} \int_{0}^{\infty} f(y)\left[\mathcal{C}\left(k_{n}\right)(x+y)+\mathcal{C}\left(k_{n}\right)(|x-y|)\right] d y \\
& =\frac{\pi}{2} \int_{0}^{\infty} f(y)\left[g_{n}(x+y)+g_{n}(|x-y|)\right] d y \\
& =\pi\left(f *_{c} g_{n}\right)(x) .
\end{aligned}
$$

Since $\left(g_{n}\right)$ satisfies the properties $(P 1),(P 2)$ and $(P 3)$ in the hypothesis of Theorem 2.10 (see [3, pp.137-138]), we get $f_{n}=\pi\left(f *_{c} g_{n}\right) \rightarrow \pi f$ as $n \rightarrow \infty$ in $L^{1}([0, \infty))$.

Definition 2.12. ([11]) For $f, g \in L^{1}([0, \infty))$, the Fourier sine convolution $*_{s c}$ is defined by $\left(f *_{s c} g\right)(t)=\frac{1}{2} \int_{0}^{\infty} f(s)[g(|t-s|)-g(t+s)] d s, \forall t \geq 0$.

Lemma 2.13. For every $f, g, h \in L^{1}([0, \infty))$ and $\alpha, \beta \in \mathbb{C},(\alpha f+\beta g) *_{s c} h=$ $\alpha\left(f *_{s c} h\right)+\beta\left(g *_{s c} h\right)$.

Lemma 2.14. If $f, g, h \in L^{1}([0, \infty))$, then $\left(f *_{s c} g\right) *_{s c} h=f *_{s c}\left(g *_{c} h\right)$.

Proof. For $x \geq 0$,

$$
\begin{aligned}
4\left[\left(f *_{s c} g\right) *_{s c} h\right](x) \\
=2 \int^{\infty}\left(f *_{s c} g\right)(y)[h(|x-y|)-h(x+y)] d y \\
=\int_{0}^{\infty}\left(\int_{0}^{\infty} f(z)[g(|y-z|)-g(y+z)] d z\right)[h(|x-y|)-h(x+y)] d y \\
=\int_{0}^{\infty} f(z) \int_{0}^{\infty}[g(|y-z|)-g(y+z)][h(|x-y|)-h(x+y)] d y d z \\
=\quad \int_{0}^{\infty} f(z)\left(\int_{0}^{\infty} g(|y-z|)[h(|x-y|)-h(x+y)] d y\right. \\
\left.\quad-\int_{0}^{\infty} g(y+z)[h(|x-y|)-h(x+y)] d y\right) d z \\
\quad \int_{0}^{\infty} f(z)\left(\int_{-z}^{\infty} g(|s|)[h(|x-z-s|)-h(x+z+s)] d s\right. \\
\left.\quad-\int_{z}^{\infty} g(t)[h(|x-t+z|)-h(x+t-z)] d t\right) d z
\end{aligned}
$$

(using the change of variables $s=y-z$ in the first integral and $t=y+z$ in the second integral) 


$$
\begin{aligned}
& =\int_{0}^{\infty} f(z)\left(\int_{-z}^{0} g(-s)[h(|x-z-s|)-h(x+z+s)] d s\right. \\
& +\int_{0}^{\infty} g(s)[h(|x-z-s|)-h(x+z+s)] d s \\
& \left.-\int_{z}^{\infty} g(t)[h(|x+z-t|)-h(x-z+t)] d t\right) d z \\
& =\int_{0}^{\infty} f(z)\left(\int_{0}^{z} g(s)[h(|x-z+s|)-h(x+z-s)] d s\right. \\
& +\int_{0}^{\infty} g(s)[h(|x-z-s|)-h(x+z+s)] d s \\
& \left.-\int_{z}^{\infty} g(t)[h(|x+z-t|)-h(x-z+t)] d t\right) d z \\
& =\int_{0}^{\infty} f(z)\left(\int_{0}^{z} g(s)[h(|x-z+s|)-h(|x+z-s|)] d s\right. \\
& +\int_{0}^{\infty} g(s)[h(|x-z-s|)-h(x+z+s)] d s \\
& \left.+\int_{z}^{\infty} g(t)[-h(|x+z-t|)+h(|x-z+t|)] d t\right) d z \\
& =\int_{0}^{\infty} f(z)\left(\int_{0}^{\infty} g(s)[h(|x-z+s|)-h(|x+z-s|)] d s\right. \\
& \left.+\int_{0}^{\infty} g(s)[h(|x-z-s|)-h(x+z+s)] d s\right) d z \\
& =\int_{0}^{\infty} f(z) \int_{0}^{\infty} g(s)[h(|x-z+s|)-h(|x+z-s|)+h(|x-z-s|) \\
& =\int_{0}^{\infty} f(z)\left(\int_{0}^{\infty} g(s)[h(|x-z+s|)+h(|x-z-s|)] d s\right. \\
& \left.-\int_{0}^{\infty} f(z) \int_{0}^{\infty} g(s)[h(|x+z-s|)+h(x+z+s)] d s\right) d z \\
& =\int_{0}^{\infty} f(z)\left(\int_{0}^{\infty} g(s)[h(|| x-z|+s|)+h(|| x-z|-s|)] d s\right. \\
& \left.-\int_{0}^{\infty} f(z) \int_{0}^{\infty} g(s)[h(|x+z-s|)+h(x+z+s)] d s\right) d z \\
& (\text { since } h(|| x-z|+s|)+h(|| x-z|-s|) \\
& = \begin{cases}h(|x-z+s|)+h(|x-z-s|) & \text { if } x-z \geq 0 \\
h(|z-x+s|)+h(|z-x-s|) & \text { if } x-z<0\end{cases} \\
& \text { and } h(|z-x+s|)+h(|z-x-s|)=h(|x-z-s|)+h(|x-z+s|)) \\
& =2 \int_{0}^{\infty} f(z)\left(\left(g *_{c} h\right)(|x-z|)-\left(g *_{c} h\right)(x+z)\right) d z \\
& =4\left[f *_{s c}\left(g *_{c} h\right)\right](x) \text {. }
\end{aligned}
$$


Theorem 2.15. (Convolution theorem for Fourier sine transform on $\left.L^{1}([0, \infty))\right)$ If $f, g \in L^{1}([0, \infty))$, then $\mathcal{S}\left(f *_{s c} g\right)=\mathcal{S}(f) \cdot \mathcal{C}(g)$.

Lemma 2.16. For $f, g \in L^{1}([0, \infty)),\left\|f *_{s c} g\right\|_{1} \leq\|f\|_{1}\|g\|_{1}$ and $f_{n} *_{s c} g \rightarrow f *_{s c} g$ as $n \rightarrow \infty$ in $L^{1}([0, \infty))$, whenever $f_{n} \rightarrow f$ as $n \rightarrow \infty$ in $L^{1}([0, \infty))$.

Proof. For $f, g \in L^{1}([0, \infty))$, we have

$$
\begin{aligned}
\left\|f *_{s c} g\right\|_{1} & =\int_{0}^{\infty}\left|\left(f *_{s c} g\right)(s)\right| d s \\
& =\frac{1}{2} \int_{0}^{\infty}\left|\int_{0}^{\infty} f(t)[g(|s-t|)-g(s+t)] d t\right| d s \\
& \leq \frac{1}{2} \int_{0}^{\infty} \int_{0}^{\infty}|f(t)||g(|s-t|)-g(s+t)| d t d s \\
& =\frac{1}{2} \int_{0}^{\infty}|f(t)| \int_{0}^{\infty}|g(|s-t|)-g(s+t)| d s d t \\
& \leq \frac{1}{2} \int_{0}^{\infty}|f(t)|\left(\int_{-t}^{\infty}|g(|u|)| d u+\int_{t}^{\infty}|g(u)| d u\right) d t \\
& =\frac{1}{2} \int_{0}^{\infty}|f(t)|\left(\int_{0}^{t}|g(u)| d u+\int_{0}^{\infty}|g(u)| d u+\int_{t}^{\infty}|g(u)| d u\right) d t \\
& =\|f\|_{1}\|g\|_{1} .
\end{aligned}
$$

Let $f_{n} \rightarrow f$ as $n \rightarrow \infty$ in $L^{1}([0, \infty))$. Using Lemma 2.13 , we get $\left\|f_{n} *_{s c} g-f *_{s c} g\right\|_{1}=\left\|\left(f_{n}-f\right) *_{s c} g\right\|_{1} \leq\left\|f_{n}-f\right\|_{1}\|g\|_{1} \rightarrow 0$ as $n \rightarrow \infty$.

Theorem 2.17. Under the hypothesis of Theorem 2.10, we have $f *_{s c} g_{n} \rightarrow f$ as $n \rightarrow \infty$ in $L^{1}([0, \infty))$.

Proof. Let $\epsilon>0$. For each $n \in \mathbb{N}$, define $\psi_{n}(s)=|f(s)| \int_{s}^{\infty}\left|g_{n}(t)\right| d t, \forall s \in(0, \infty)$. Then, using the property $(P 3)$ of $\left(g_{n}\right)$, we have, for each $s>0, \lim _{n \rightarrow \infty} \psi_{n}(s)=$ $\lim _{n \rightarrow \infty}|f(s)| \int_{s}^{\infty}\left|g_{n}(t)\right| d t=0$ and from $(P 2)$, there exists $M>0$ such that $\left|\psi_{n}(s)\right| \leq$ $|f(s)| \int_{0}^{\infty}\left|g_{n}(t)\right| d t \leq M|f(s)| \in L^{1}([0, \infty)), \forall n \in \mathbb{N}$. Applying Lebesgue dominated convergence theorem, we get $\int_{0}^{\infty} \psi_{n}(s) d s \rightarrow 0$ as $n \rightarrow \infty$. Now for each $n \in \mathbb{N}$, we have

$$
\begin{aligned}
\left(f *_{s c} g_{n}\right)(x)-f(x) & =\frac{1}{2} \int_{0}^{\infty} f(y)\left[g_{n}(|x-y|)-g_{n}(x+y)\right] d y-f(x) \\
& =\frac{1}{2} \int_{0}^{\infty} f(y)\left[g_{n}(|x-y|)+g_{n}(x+y)-2 g_{n}(x+y)\right] d y-f(x) \\
& =\left(f *_{c} g_{n}\right)(x)-f(x)-\int_{0}^{\infty} f(y) g_{n}(x+y) d y
\end{aligned}
$$

Therefore, 


$$
\begin{aligned}
\left\|\left(f *_{s c} g_{n}\right)-f\right\|_{1} \leq & \left\|\left(f *_{c} g_{n}\right)-f\right\|_{1}+\int_{0}^{\infty}\left|\int_{0}^{\infty} f(y) g_{n}(x+y) d y\right| d x \\
\leq & \left\|\left(f *_{c} g_{n}\right)-f\right\|_{1}+\int_{0}^{\infty} \int_{0}^{\infty}\left|f(y) \| g_{n}(x+y)\right| d y d x \\
= & \left\|\left(f *_{c} g_{n}\right)-f\right\|_{1}+\int_{0}^{\infty}|f(y)| \int_{0}^{\infty}\left|g_{n}(x+y)\right| d x d y \\
\leq\left\|\left(f *_{c} g_{n}\right)-f\right\|_{1}+\int_{0}^{\infty}|f(y)| \int_{y}^{\infty}\left|g_{n}(u)\right| d u d y & \quad\left\|\left(f *_{c} g_{n}\right)-f\right\|_{1}+\int_{0}^{\infty} \psi_{n}(y) d y
\end{aligned}
$$

which tends to zero as $n \rightarrow \infty$, by using Theorem 2.10 .

Now, we present a characterization theorem for the range space of the Fourier sine transform on $L^{1}([0, \infty))$.

Theorem 2.18. Let $F \in C_{0}([0, \infty))$. Then, $F \in \mathcal{S}\left(L^{1}([0, \infty))\right)$ if and only if $\left(f_{n}\right)$ converges in $L^{1}([0, \infty))$, where

$$
f_{n}(x)=\int_{0}^{n}\left(1-\frac{t}{n}\right) F(t) \sin (x t) d t, \forall x \in[0, \infty) \text { and } n=1,2,3, \cdots
$$

Proof. Suppose that $f_{n} \rightarrow f$ as $n \rightarrow \infty$ in $L^{1}([0, \infty))$, for some $f \in L^{1}([0, \infty))$. If $h_{n}(t)=\chi_{[0, n]}(t)\left(1-\frac{t}{n}\right) F(t), \forall t \in[0, \infty), \forall n \in \mathbb{N}$, then we have $\mathcal{S}\left(f_{n}\right)=\mathcal{S}\left[\mathcal{S}\left(h_{n}\right)\right]=$ $h_{n}, \forall n \in \mathbb{N}$. Using the continuity of $\mathcal{S}$, we get $\mathcal{S}(f)=\lim _{n \rightarrow \infty} \mathcal{S}\left(f_{n}\right)=\lim _{n \rightarrow \infty} h_{n}=F$.

Conversely, assume that there exists $f \in L^{1}([0, \infty))$ such that $F=\mathcal{S}(f)$.

Now following the notations in the proof of Theorem 2.11, we get

$$
\begin{aligned}
f_{n}(x)= & \int_{0}^{n}\left(1-\frac{t}{n}\right) F(t) \sin (x t) d t=\int_{0}^{n}\left(1-\frac{t}{n}\right)\left(\int_{0}^{\infty} f(y) \sin (y t) d y\right) \sin (x t) d t \\
= & \frac{1}{2} \int_{0}^{\infty} f(y)\left(\int_{0}^{n}\left(1-\frac{t}{n}\right)[\cos (|x-y| t)-\cos ((x+y) t)] d t\right) d y \\
& \quad \text { using Fubini's theorem }) \\
= & \frac{1}{2} \int_{0}^{\infty} f(y)\left[\mathcal{C}\left(k_{n}\right)(|x-y|)-\mathcal{C}\left(k_{n}\right)(x+y)\right] d y,
\end{aligned}
$$

Since $\left(g_{n}\right)=\left(\pi^{-1} \mathcal{C}\left(k_{n}\right)\right)$ satisfies properties $(P 1),(P 2)$ and $(P 3)$, applying Theorem 2.17 , we obtain that $f_{n}=\pi\left(f *_{s c} g_{n}\right) \rightarrow \pi f$ as $n \rightarrow \infty$ in $L^{1}([0, \infty))$.

\section{Boehmian Spaces}

Before starting this section, we briefly recall the general construction of Boehmian space and two notions of convergence in the context of Boehmian space from the literature $[6,9]$.

Let $G$ be a topological vector space, $(S, *)$ be a commutative semi-group, * $G \times S \rightarrow G$ satisfy the following conditions. 
(i) If $f, g \in G$ and $\psi \in S$, then $(f+g) \star \psi=(f \star \psi)+(g \star \psi)$,

(ii) If $f \in G, \phi \in S$ and $\alpha \in \mathbb{C}$, then $(\alpha f) \star \phi=\alpha(f \star \phi)$,

(iii) If $f \in G$ and $\varphi, \psi \in S$, then $(f \star \varphi) \star \psi=f \star(\varphi * \psi)$.

Let $\Delta$ be a collection of sequences from $S$ with the following properties.

(a) If $f_{n} \rightarrow f$ as $n \rightarrow \infty$ in $G$ and $\left(\varphi_{n}\right) \in \Delta, f_{n} \star \varphi_{n} \rightarrow f$ as $n \rightarrow \infty$,

(b) If $\left(\varphi_{n}\right),\left(\psi_{n}\right) \in \Delta$, then $\left(\varphi_{n} * \psi_{n}\right) \in \Delta$.

Let $\mathcal{A}=\left\{\left\{\left(f_{n}\right),\left(\varphi_{n}\right)\right\}: f_{n} \in G,\left(\varphi_{n}\right) \in \Delta\right.$, and $f_{n} \star \varphi_{m}=f_{m} \star \varphi_{n}, \forall n, m \in$ $\mathbb{N}\}$. An equivalence relation $\sim$ on $\mathcal{A}$ is defined by $\left\{\left(f_{n}\right),\left(\varphi_{n}\right)\right\} \sim\left\{\left(g_{n}\right),\left(\psi_{n}\right)\right\}$ if $f_{n} \star \psi_{m}=g_{m} \star \varphi_{n}, \forall n, m \in \mathbb{N}$. The collection of all equivalence classes induced by $\sim$ on $\mathcal{A}$ is called the Boehmian space $\mathcal{B}=\mathcal{B}(G,(S, *), \star, \Delta)$ and any element of $\mathcal{B}$ is denoted by $X=\left[\frac{\left(f_{n}\right)}{\left(\varphi_{n}\right)}\right]$. We identify $G$ as a subset of $\mathcal{B}$, through the identification $f \mapsto\left[\frac{\left(f * \varphi_{n}\right)}{\left(\varphi_{n}\right)}\right]$, where $\left(\varphi_{n}\right) \in \Delta$ is arbitrary. For $X=\left[\frac{\left(f_{n}\right)}{\left(\varphi_{n}\right)}\right], Y=\left[\frac{\left(g_{n}\right)}{\left(\psi_{n}\right)}\right] \in \mathcal{B}$, $\alpha \in \mathbb{C}$ and $\eta \in S$, we also define $X+Y=\left[\frac{\left(f_{n} \star \psi_{n}+g_{n} \star \varphi_{n}\right)}{\left(\varphi_{n} * \psi_{n}\right)}\right], \alpha X=\left[\frac{\left(\alpha f_{n}\right)}{\left(\varphi_{n}\right)}\right]$, $X \star \eta=\left[\frac{\left(f_{n} \star \eta\right)}{\left(\varphi_{n}\right)}\right]$ and $X \star Y=\left[\frac{\left(f_{n} \star g_{n}\right)}{\left(\varphi_{n} * \psi_{n}\right)}\right]$, provided $g_{n} \in S, \forall n \in \mathbb{N}$.

Lemma 3.1. If $X=\left[\frac{\left(f_{n}\right)}{\left(\varphi_{n}\right)}\right] \in \mathcal{B}$, then $X \star \varphi_{k}=f_{k} \in G$ for all $k \in \mathbb{N}$.

Definition 3.2. A sequence $\left(X_{n}\right)$ of Boehmians is said to $\delta$-converge to $X$ in $\mathcal{B}$ (denoted by $X_{n} \stackrel{\delta}{\rightarrow} X$ as $n \rightarrow \infty$ ) if there exists $\left(\delta_{n}\right) \in \Delta$ such that $X_{n} \star \delta_{k}$, $X \star \delta_{k} \in G, \forall n, k \in \mathbb{N}$ and for each $k \in \mathbb{N}, X_{n} \star \delta_{k} \rightarrow X \star \delta_{k}$ as $n \rightarrow \infty$ in $G$.

Theorem 3.3. $X_{n} \stackrel{\delta}{\rightarrow} X$ as $n \rightarrow \infty$ if and only if there exist $f_{n, k}, f_{k} \in G$ and $\left(\delta_{n}\right) \in \Delta$ such that $X_{n}=\left[\frac{\left(f_{n, k}\right)}{\left(\delta_{k}\right)}\right], X=\left[\frac{\left(f_{k}\right)}{\left(\delta_{k}\right)}\right]$ and $f_{n, k} \rightarrow f_{k}$ as $n \rightarrow \infty$ in $G$, $\forall k \in \mathbb{N}$.

Definition 3.4. A sequence $\left(X_{n}\right)$ of Boehmians $\Delta$-converges to $X$ in $\mathcal{B}$ (denoted by $X_{n} \stackrel{\Delta}{\rightarrow} X$ as $\left.n \rightarrow \infty\right)$ if there exists $\left(\delta_{n}\right) \in \Delta$ such that $\left(X_{n}-X\right) \star \delta_{n} \in G, \forall n \in \mathbb{N}$ and $\left(X_{n}-X\right) \star \delta_{n} \rightarrow 0$ as $n \rightarrow \infty$ in $\mathrm{G}$.

Now we prove some auxiliary results required to construct the Fourier cosine transformable Boehmian space $\mathcal{B}_{c}^{1}=\mathcal{B}\left(L^{1}([0, \infty)),\left(L^{1}([0, \infty)), *_{c}\right), *_{c}, \Delta_{+}\right)$, where $\Delta_{+}$is the the class of all sequences $\left(\delta_{n}\right)$ from $L^{1}([0, \infty))$ satisfying the following conditions:

(a) $\int_{0}^{\infty} \delta_{n}(s) d s=1, \forall n \in \mathbb{N}$; (b) $\int_{0}^{\infty}\left|\delta_{n}(s)\right| d s \leq M, \forall n \in \mathbb{N}$, for some $M>0$; and (c) supp $\delta_{n} \rightarrow\{0\}$ as $n \rightarrow \infty$, that is, there exists $N \in \mathbb{N}$ such that $\delta_{n}(t)=0, \forall t \geq \delta$ and $n \geq N$.

Lemma 3.5. If $f \in L^{1}([0, \infty))$ and $\left(\delta_{n}\right) \in \Delta_{+}$, then $f *_{c} \delta_{n} \rightarrow f$ as $n \rightarrow \infty$ in $L^{1}([0, \infty))$.

Proof. Let $\delta>0$ be given. By property $(c)$ of $\left(\delta_{n}\right),(P 3)$ holds true for $\left(\delta_{n}\right)$. Thus from Theorem 2.10, we get $f *_{c} \delta_{n} \rightarrow f$ as $n \rightarrow \infty$ in $L^{1}([0, \infty))$. 
Lemma 3.6. If $f_{n} \rightarrow f$ as $n \rightarrow \infty$ in $L^{1}([0, \infty))$ and $\left(\delta_{n}\right) \in \Delta_{+}$, then $f_{n} *_{c} \delta_{n} \rightarrow f$ as $n \rightarrow \infty$ in $L^{1}([0, \infty))$.

Proof. Consider $\left\|\left(f_{n} *_{c} \delta_{n}\right)-f\right\|_{1} \leq\left\|\left(f_{n}-f\right) *_{c} \delta_{n}\right\|_{1}+\left\|f *_{c} \delta_{n}-f\right\|_{1}, \forall n \in \mathbb{N}$. The second term in the right hand side of the above inequality tends to zero, by the previous lemma. Using the estimate $\left\|f *_{c} g\right\|_{1} \leq\|f\|_{1}\|g\|_{1}$ (See the proof of Lemma 2.4), we have $\left\|\left(f_{n}-f\right) *_{c} \delta_{n}\right\|_{1} \leq\left\|\left(f_{n}-f\right)\right\|_{1} \int_{0}^{\infty}\left|\delta_{n}(t)\right| d t \leq M\left\|\left(f_{n}-f\right)\right\|_{1} \rightarrow$ 0 as $n \rightarrow \infty$.

Lemma 3.7. If $\left(\delta_{n}\right),\left(\psi_{n}\right) \in \Delta_{+}$, then $\left(\delta_{n} *_{c} \psi_{n}\right) \in \Delta_{+}$.

Proof. Using Fubini's theorem, we get

$$
\begin{aligned}
\int_{0}^{\infty}\left(\delta_{n} *_{c} \psi_{n}\right)(s) d s & =\frac{1}{2} \int_{0}^{\infty} \int_{0}^{\infty}\left[\delta_{n}(s+t)+\delta_{n}(|s-t|)\right] \psi_{n}(t) d t d s \\
& =\frac{1}{2} \int_{0}^{\infty} \psi_{n}(t) \int_{0}^{\infty}\left[\delta_{n}(s+t)+\delta_{n}(|s-t|)\right] d s d t \\
& =\frac{1}{2} \int_{0}^{\infty} \psi_{n}(t)\left[\int_{t}^{\infty} \delta_{n}(u) d u+\int_{-t}^{\infty} \delta_{n}(|u|) d u\right] d t \\
& =\frac{1}{2} \int_{0}^{\infty} \psi_{n}(t)\left[\int_{t}^{\infty} \delta_{n}(u) d u+\int_{-t}^{0} \delta_{n}(-u) d u+\int_{0}^{\infty} \delta_{n}(u) d u\right] d t \\
& =\frac{1}{2} \int_{0}^{\infty} \psi_{n}(t)\left[\int_{t}^{\infty} \delta_{n}(u) d u+\int_{0}^{t} \delta_{n}(u) d u+\int_{0}^{\infty} \delta_{n}(u) d u\right] d t \\
& =\int_{0}^{\infty} \psi_{n}(t)\left[\int_{0}^{\infty} \delta_{n}(u) d u\right] d t=\int_{0}^{\infty} \psi_{n}(t) d t=1 .
\end{aligned}
$$

By a similar argument, it is easy to verify that $\int_{0}^{\infty}\left|\left(\delta_{n} *_{c} \psi_{n}\right)(s)\right| d s \leq M_{1} M_{2}$ where $M_{1}, M_{2} \in \mathbb{R}$ are such that $\int_{0}^{\infty}\left|\delta_{n}(t)\right| d t \leq M_{1}$ and $\int_{0}^{\infty}\left|\psi_{n}(t)\right| d t \leq M_{2}, \forall n \in \mathbb{N}$.

Since $\operatorname{supp}\left(\delta_{n} *_{c} \psi_{n}\right) \subset\left[\operatorname{supp} \delta_{n}+\operatorname{supp} \psi_{n}\right] \cup\left([0, \infty) \cap\left[\operatorname{supp} \delta_{n}-\operatorname{supp} \psi_{n}\right]\right) \cup$ $\left([0, \infty) \cap\left[\operatorname{supp} \psi_{n}-\operatorname{supp} \delta_{n}\right]\right)$, we get $\operatorname{supp}\left(\delta_{n} *_{c} \psi_{n}\right) \rightarrow\{0\}$ as $n \rightarrow \infty$. Hence it follows that $\left(\delta_{n} *_{c} \psi_{n}\right) \in \Delta_{+}$.

Thus the Fourier cosine transformable Boehmian space $\mathcal{B}_{c}^{1}$ is constructed.

Example 3.8. If $\phi_{n}(t)=n \chi_{\left[0, \frac{1}{n}\right]}(t), \forall t \in[0, \infty), \forall n \in \mathbb{N}$, then $\left(\phi_{n}\right) \in \Delta_{+}$and hence $\mathcal{C}\left(\phi_{n}\right) \rightarrow 1$, uniformly on compact subsets of $[0, \infty)$, which will be proved later in Lemma 4.1. Since $*_{c}$ is commutative, we have $\phi_{m} *_{c} \phi_{n}=\phi_{n} *_{c} \phi_{m}, \forall m, n \in \mathbb{N}$. That is $\left[\frac{\left(\phi_{n}\right)}{\left(\phi_{n}\right)}\right] \in \mathcal{B}_{c}^{1}$. From the definition of $\mathfrak{C}$ on $\mathcal{B}_{c}^{1}$, it is clear that $\mathfrak{C}(X)=\lim _{n \rightarrow \infty} \mathfrak{C}\left(\phi_{n}\right)=$ 1 (see Definition 4.2). Since $1 \in C([0, \infty)) \backslash C_{0}([0, \infty))$, this Boehmian does not represent any element of $L^{1}([0, \infty))$.

Next, we prove the auxiliary results required to construct another Boehmian space $\mathcal{B}_{s}^{1}=\mathcal{B}\left(L^{1}([0, \infty)),\left(L^{1}([0, \infty)), *_{c}\right), *_{s c}, \Delta_{+}\right)$.

Lemma 3.9. If $f \in L^{1}([0, \infty))$ and $\left(\delta_{n}\right) \in \Delta_{+}$, then $f *_{s c} \delta_{n} \rightarrow f$ as $n \rightarrow \infty$ in 
$L^{1}([0, \infty))$

Proof. Since $\left(\delta_{n}\right) \in \Delta_{+}$satisfies $(P 1),(P 2)$ and $(P 3)$, the proof directly follows from Theorem 2.17 .

Lemma 3.10. If $f_{n} \rightarrow f$ as $n \rightarrow \infty$ in $L^{1}([0, \infty))$ and $\left(\delta_{n}\right) \in \Delta_{+}$, then $f_{n} *_{s c} \delta_{n} \rightarrow f$ as $n \rightarrow \infty$ in $L^{1}([0, \infty))$.

Proof. Using Lemma 2.13, Lemma 2.4 and Lemma 3.9, we obtain the proof of this lemma which is similar to that of Lemma 3.6.

Thus, we constructed the Fourier sine transformable Boehmian space $\mathcal{B}_{s}^{1}$.

Example 3.11. Define $f(x)=\left\{\begin{array}{cc}1-\frac{1}{1+x} & \text { for } x \geq 0 \\ 0 & \text { for } x<0 .\end{array}\right.$ Then $f$ is bounded, $f(0)=0$ and $f \notin L^{1}([0, \infty))$ but $f^{\prime}(x) \in L^{1}([0, \infty))$. Choose a smooth function $\psi$ with compact support on $[0, \infty)$ such that $\int_{0}^{\infty} \psi^{\prime}(t) d t=1$. If $\psi_{n}(x)=n \psi^{\prime}(n x), \forall x \in$ $[0, \infty), \forall n \in \mathbb{N}$, then $\left(\psi_{n}\right) \in \Delta_{+}$. We claim that $X=\left[\frac{f *_{s} \psi_{n}}{\psi_{n}}\right] \in \mathcal{B}_{s}^{1} \backslash L^{1}([0, \infty))$.

To justify the example, we first claim that $f *_{s c} \psi_{n} \in L^{1}([0, \infty)), \forall n \in \mathbb{N}$.

Since $f(0)=\psi(0)=0$ and $f$ is bounded, we have

$$
\begin{aligned}
\mid\left(f *_{s c}\right. & \left.\psi_{n}\right)(x) \mid \\
= & \frac{1}{2}\left|\int_{0}^{\infty} f(y) \psi_{n}(|x-y|) d y-\int_{0}^{\infty} f(y) \psi_{n}(x+y) d y\right| \\
= & \frac{1}{2}\left|\int_{0}^{x} f(y) \psi_{n}(x-y) d y+\int_{x}^{\infty} f(y) \psi_{n}(y-x) d y-\int_{0}^{\infty} f(y) \psi_{n}(x+y) d y\right| \\
= & \frac{1}{2}\left|\int_{0}^{x} f(y) d[-\psi(n(x-y))]+\int_{x}^{\infty} f(y) d[\psi(n(y-x))]-\int_{0}^{\infty} f(y) d[\psi(n(x+y))]\right| \\
= & \frac{1}{2} \mid[-f(y) \psi(n(x-y))]_{0}^{x}+\int_{0}^{x} f^{\prime}(y) \psi(n(x-y)) d y+[f(y) \psi(n(y-x))]_{x}^{\infty} \\
= & -\int_{x}^{\infty} f^{\prime}(y) \psi(n(y-x)) d y+[f(y) \psi(n(x+y))]_{0}^{\infty}-\int_{0}^{\infty} f^{\prime}(y) \psi(n(x+y)) d y \mid \\
\leq & \frac{1}{2}\left(\int_{0}^{\infty}\left|f^{\prime}(y) \psi(n(x-y)) d y-\int_{x}^{\infty} f^{\prime}(y) \psi(n(y-x)) d y-\int_{0}^{\infty} f^{\prime}(y) \psi(n(x+y)) d y\right|\right. \\
& \left.+\int_{0}^{\infty}\left|f^{\prime}(y)\right||\psi(n(x+y))| d y\right)\left|d y+\int_{x}^{\infty}\right| f^{\prime}(y)|| \psi(n(y-x)) \mid d y \\
= & \frac{1}{2}\left(\int_{0}^{\infty}\left|f^{\prime}(y)\right||\psi(n|x-y|)| d y+\int_{0}^{\infty}\left|f^{\prime}(y)\right||\psi(n(x+y))| d y\right) .
\end{aligned}
$$

Using Fubini's theorem, we get

$\left\|f *_{s c} \psi_{n}\right\|_{1}$ 


$$
\begin{aligned}
& \leq \frac{1}{2}\left(\int_{0}^{\infty}\left|f^{\prime}(y)\right| \int_{0}^{\infty}|\psi(n|x-y|)| d x d y+\int_{0}^{\infty}\left|f^{\prime}(y)\right| \int_{0}^{\infty}|\psi(n(x+y))| d x d y\right) \\
& \leq \frac{1}{2}\left(\int_{0}^{\infty}\left|f^{\prime}(y)\right| \int_{-y}^{\infty}|\psi(n|s|)| d s d y+\int_{0}^{\infty}\left|f^{\prime}(y)\right| \int_{y}^{\infty}|\psi(n s)| d s d y\right) \\
& =\int_{0}^{\infty}\left|f^{\prime}(y)\right| \int_{0}^{\infty}|\psi(n s)| d s d y<+\infty
\end{aligned}
$$

Applying similar arguments as in the proof of Lemma 2.14 and using Lemma 2.6 , we get $\left(f *_{s c} \psi_{n}\right) *_{s c} \psi_{m}=f *_{s c}\left(\psi_{n} *_{c} \psi_{m}\right)=f *_{s c}\left(\psi_{m} *_{c} \psi_{n}\right)=\left(f *_{s c} \psi_{m}\right) *_{s c}$ $\psi_{n}, \forall m, n \in \mathbb{N}$. Thus, we obtain that $X \in \mathcal{B}_{s}^{1}$ and by the choice of $f, X$ does not represent any function in $L^{1}([0, \infty))$.

\section{Extended Fourier Cosine and Sine Transforms}

First, we prove the following lemma which will be required to define the extended Fourier cosine and sine transforms.

Lemma 4.1. If $\left(\delta_{n}\right) \in \Delta_{+}$, then $\mathrm{C}\left(\delta_{n}\right) \rightarrow 1$ as $n \rightarrow \infty$ uniformly on compact subset of $[0, \infty)$.

Proof. Let $K$ be a compact subset of $[0, \infty)$. Let $\epsilon>0$ be given. Choose $\Lambda>0, M>0$ and a positive integer $N$ such that $t \leq \Lambda$ for all $t \in K$, $\int_{0}^{\infty}\left|\delta_{n}(t)\right| d t \leq M, \forall n \in \mathbb{N}$ and $\operatorname{supp} \delta_{n} \subset[0, \epsilon)$ for all $n \geq N$. For $t \in K$ and $n \geq N$, applying mean-value theorem, we get

$$
\begin{aligned}
\left|\mathcal{C}\left(\delta_{n}\right)(t)-1\right| & =\left|\int_{0}^{\infty} \delta_{n}(s) \cos (t s) d s-\int_{0}^{\infty} \delta_{n}(s) d s\right| \leq \int_{0}^{\infty}\left|\delta_{n}(s)\right||\cos (t s)-1| d s \\
& =\int_{0}^{\epsilon}\left|\delta_{n}(s)\right||\cos (t s)-1| d s \quad=\int_{0}^{\epsilon}\left|\delta_{n}(s)\right||t s||\sin z| d s \\
& \leq \Lambda \epsilon \int_{0}^{\epsilon}\left|\delta_{n}(s)\right| d s \leq \Lambda \epsilon \int_{0}^{\infty}\left|\delta_{n}(s)\right| d s \leq \Lambda M \epsilon .
\end{aligned}
$$

This completes the proof.

Definition 4.2. For $X=\left[\frac{\left(f_{n}\right)}{\left(\delta_{n}\right)}\right] \in \mathcal{B}_{c}^{1}$, we define the extended Fourier cosine transform $\mathfrak{C}(X)$ by

$$
\mathfrak{C}(X)(t)=\lim _{n \rightarrow \infty} \mathcal{C}\left(f_{n}\right)(t), \forall t \in[0, \infty)
$$

Note that the above limit exists. Indeed, given a compact subset $K$ of $[0, \infty)$, some $k \in \mathbb{N}$ can be chosen so that $\mathcal{C}\left(\delta_{k}\right) \neq 0$ on $K$, because $\mathcal{C}\left(\delta_{n}\right) \rightarrow 1$ as $n \rightarrow \infty$ in $C([0, \infty))$. Then, from Theorem 2.8, it follows that $\mathcal{C}\left(f_{n}\right)=$

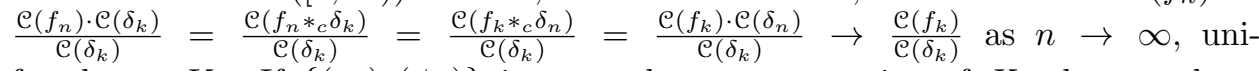
formly on $K$. If $\left\{\left(g_{n}\right),\left(\psi_{n}\right)\right\}$ is any other representative of $X$, then we have $\mathcal{C}\left(f_{n}\right)(s) \cdot \mathcal{C}\left(\psi_{m}\right)(s)=\mathcal{C}\left(g_{m}\right)(s) \cdot \mathcal{C}\left(\delta_{n}\right)(s)$ for all $m, n \in \mathbb{N}$ and $s \geq 0$. Now for a given $t \in[0, \infty)$, choose $m, k \in \mathbb{N}$ such that $\mathcal{C}\left(\psi_{m}\right)(t) \neq 0 \neq \mathcal{C}\left(\delta_{k}\right)(t)$. Then, $\lim _{n \rightarrow \infty} \mathcal{C}\left(g_{n}\right)(t)=\frac{\mathfrak{C}\left(g_{m}\right)(t)}{\mathcal{C}\left(\psi_{m}\right)(t)}=\frac{\mathfrak{C}\left(f_{k}\right)(t)}{\mathcal{C}\left(\delta_{k}\right)(t)}=\lim _{n \rightarrow \infty} \mathcal{C}\left(f_{n}\right)(t)$. Thus the above 
limit is independent of the choice of representatives of $X$. Further, by using the fact that $\mathcal{C}\left(f_{n}\right)$ converges uniformly on compact subsets of $[0, \infty)$, we have $\mathfrak{C}(X)=\lim _{n \rightarrow \infty} \mathcal{C}\left(f_{n}\right) \in C([0, \infty))$.

Theorem 4.3. The extended Fourier cosine transform $\mathfrak{C}: \mathcal{B}_{c}^{1} \rightarrow C([0, \infty))$ is consistent with $\mathcal{C}: L^{1}([0, \infty)) \rightarrow C_{0}([0, \infty))$.

Proof. Let $f \in L^{1}([0, \infty))$. Then for any $\left(\delta_{n}\right) \in \Delta$, the Boehmian $\left[\frac{\left(f *_{c} \delta_{n}\right)}{\left(\delta_{n}\right)}\right]$ is representing $f$ in $\mathcal{B}_{c}^{1}$. Then $\mathfrak{C}\left(\left[\frac{\left(f *_{c} \delta_{n}\right)}{\left(\delta_{n}\right)}\right]\right)=\lim _{n \rightarrow \infty} \mathcal{C}\left(f *_{c} \delta_{n}\right)=\lim _{n \rightarrow \infty} \mathcal{C}(f) \cdot \mathcal{C}\left(\delta_{n}\right)=$ $\mathcal{C}(f)$, since $\mathrm{C}\left(\delta_{n}\right) \rightarrow 1$ as $n \rightarrow \infty$. Hence the theorem follows.

Lemma 4.4. If $X=\left[\frac{\left(f_{n}\right)}{\left(\delta_{n}\right)}\right] \in \mathcal{B}_{c}^{1}$, then $\mathcal{C}\left(\delta_{k}\right) \cdot \mathfrak{C}(X)=\mathcal{C}\left(f_{k}\right), \forall k \in \mathbb{N}$.

Proof. Fix $k \in \mathbb{N}$ and $t \geq 0$, arbitrarily. If $\mathcal{C}\left(\delta_{k}\right)(t) \neq 0$, then by definition, $\mathfrak{C}(X)(t)=\frac{\mathfrak{C}\left(f_{k}\right)(t)}{\mathfrak{C}\left(\delta_{k}\right)(t)}$. This implies immediately that $\mathcal{C}\left(\delta_{k}\right)(t) \cdot \mathfrak{C}(X)(t)=\mathcal{C}\left(f_{k}\right)(t)$. If $\mathcal{C}\left(\delta_{k}\right)(t)=0$, choose $m$ so large that $\mathcal{C}\left(\delta_{m}\right)(t) \neq 0$. Since $\left[\frac{\left(f_{n}\right)}{\left(\delta_{n}\right)}\right] \in \mathcal{B}_{c}^{1}$, we have $f_{k} *_{c} \delta_{m}=f_{m} *_{c} \delta_{k}$. Applying Fourier cosine transform on both sides, we obtain that $\mathcal{C}\left(f_{k}\right) \cdot \mathcal{C}\left(\delta_{m}\right)=\mathcal{C}\left(f_{m}\right) \cdot \mathcal{C}\left(\delta_{k}\right)$ and hence $\mathcal{C}\left(f_{k}\right)(t)=\frac{\mathcal{C}\left(f_{m}\right)(t) \cdot \mathcal{C}\left(\delta_{k}\right)(t)}{\mathcal{C}\left(\delta_{m}\right)(t)}=0=$ $\mathcal{C}\left(\delta_{k}\right)(t) \cdot \mathcal{C}(X)(t)$.

Theorem 4.5. The extended Fourier cosine transform $\mathfrak{C}: \mathcal{B}_{c}^{1} \rightarrow C([0, \infty))$ is linear.

The proof of this theorem is straightforward.

Theorem 4.6. The extended Fourier cosine transform $\mathfrak{C}: \mathcal{B}_{c}^{1} \rightarrow C([0, \infty))$ is one-to-one.

Proof. Assume that $X=\left[\frac{\left(f_{n}\right)}{\left(\delta_{n}\right)}\right], Y=\left[\frac{\left(g_{n}\right)}{\left(\psi_{n}\right)}\right] \in \mathcal{B}_{c}^{1}$ are such that $\mathfrak{C}(X)(t)=\mathfrak{C}(Y)(t)$ for all $t \in[0, \infty)$. From Lemma 4.4, we have $\mathcal{C}\left(\delta_{n}\right)(t) \cdot \mathfrak{C}(X)(t)=\mathcal{C}\left(f_{n}\right)(t)$ and $\mathcal{C}\left(\psi_{n}\right)(t) \cdot \mathfrak{C}(Y)(t)=\mathcal{C}\left(g_{n}\right)(t), \forall n \in \mathbb{N}$ and $\forall t \in[0, \infty)$. Thus for $n, m \in \mathbb{N}$ and $t \in[0, \infty)$, we have $\mathcal{C}\left(g_{m} *_{c} \delta_{n}\right)(t)=\mathcal{C}\left(\delta_{n}\right)(t) \cdot \mathcal{C}\left(g_{m}\right)(t)=\mathcal{C}\left(\delta_{n}\right)(t) \cdot \mathcal{C}\left(\psi_{m}\right)(t)$. $\mathfrak{C}(Y)(t)=\mathcal{C}\left(\psi_{m}\right)(t) \cdot \mathcal{C}\left(\delta_{n}\right)(t) \cdot \mathfrak{C}(X)(t)=\mathcal{C}\left(\psi_{m}\right)(t) \cdot \mathcal{C}\left(f_{n}\right)(t)=\mathcal{C}\left(f_{n} *_{c} \psi_{m}\right)(t)$. Since $\mathcal{C}: L^{1}([0, \infty)) \rightarrow C_{0}([0, \infty))$ is one-to-one, we get $f_{n} *_{c} \psi_{m}=g_{m} *_{c} \delta_{n}$ and hence $X=Y$.

Theorem 4.7. (Convolution theorem) If $X, Y \in \mathcal{B}_{c}^{1}$ and $h \in L^{1}([0, \infty))$, then $\mathfrak{C}\left(X *_{c} h\right)=\mathfrak{C}(X) \cdot \mathfrak{C}(h)$ and $\mathfrak{C}\left(X *_{c} Y\right)=\mathfrak{C}(X) \cdot \mathfrak{C}(Y)$.

Proof. Let $X=\left[\frac{\left(f_{n}\right)}{\left(\delta_{n}\right)}\right], Y=\left[\frac{\left(g_{n}\right)}{\left(\psi_{n}\right)}\right] \in \mathcal{B}_{c}^{1}$ and $h \in L^{1}([0, \infty))$. Then we have $\mathfrak{C}\left(X *_{c} h\right)=\lim _{n \rightarrow \infty} \mathfrak{C}\left(f_{n} *_{c} h\right)=\lim _{n \rightarrow \infty}\left(\mathcal{C}\left(f_{n}\right) \cdot \mathcal{C}(h)\right)=\left(\lim _{n \rightarrow \infty} \mathcal{C}\left(f_{n}\right)\right) \cdot \mathcal{C}(h)=\mathfrak{C}(X) \cdot \mathcal{C}(h)$ and $\mathfrak{C}\left(X *_{c} Y\right) \stackrel{n \rightarrow \infty}{=} \lim _{n \rightarrow \infty} \mathcal{C}\left(f_{n} *_{c} g_{n}\right)=\lim _{n \rightarrow \infty}\left(\mathcal{C}\left(f_{n}\right) \cdot \mathcal{C}\left(g_{n}\right)\right)=\mathfrak{C}(X) \cdot \mathfrak{C}(Y)$.

Theorem 4.8. The extended Fourier cosine transform $\mathfrak{C}: \mathcal{B}_{c}^{1} \rightarrow C([0, \infty))$ is continuous with respect to $\delta$-convergence and $\Delta$-convergence. 
Proof. Let $X_{n} \stackrel{\delta}{\rightarrow} X$ as $n \rightarrow \infty$ in $\mathcal{B}_{c}^{1}$. Then, by Theorem 3.3, there are functions $f_{n, m}, f_{m} \in L^{1}([0, \infty))$ and $\left(\delta_{m}\right) \in \Delta_{+}$such that $X_{n}=\left[\frac{\left(f_{n, m}\right)}{\left(\delta_{m}\right)}\right], X=\left[\frac{\left(f_{m}\right)}{\left(\delta_{m}\right)}\right]$ and $f_{n, k} \rightarrow f_{k}$ as $n \rightarrow \infty$ in $L^{1}([0, \infty))$, for each $k \in \mathbb{N}$. Now the continuity of $\mathcal{C}: L^{1}[0, \infty) \rightarrow C_{0}([0, \infty))$ implies that $\mathrm{e}\left(f_{n, k}\right) \rightarrow \mathcal{C}\left(f_{k}\right)$ as $n \rightarrow \infty$ uniformly on $[0, \infty)$. Let $H$ be a compact subset of $[0, \infty)$. Choose $r>0$ and $k \in \mathbb{N}$ such that $0<r<\left|\mathfrak{C}\left(\delta_{n}\right)\right|$ on $H, \forall n \geq k$. Now $\left|\mathfrak{C}\left(X_{n}\right)-\mathfrak{C}(X)\right|=\frac{\left|\mathcal{C}\left(f_{n, k}\right)-\mathcal{C}\left(f_{k}\right)\right|}{\left|\mathcal{C}\left(\delta_{k}\right)\right|} \leq$ $\frac{\left|\mathcal{C}\left(f_{n, k}\right)-\mathcal{C}\left(f_{k}\right)\right|}{r} \rightarrow 0$ uniformly on $H$ as $n \rightarrow \infty$.

Let $X_{n} \stackrel{\Delta}{\rightarrow} X$ as $n \rightarrow \infty$. Then there exists $\left(\delta_{n}\right) \in \Delta_{+}$such that $\left(X_{n}-X\right) *_{c} \delta_{n} \in$ $L^{1}([0, \infty))$ and $\left(X_{n}-X\right) *_{c} \delta_{n} \rightarrow 0$ as $n \rightarrow \infty$ in $L^{1}([0, \infty))$. Let $H$ and $r$ be as in the previous argument. As the Fourier cosine transform is continuous from $L^{1}([0, \infty))$ into $C_{0}([0, \infty))$, we have $\left|\mathfrak{C}\left[\left(X_{n}-X\right) *_{c} \delta_{n}\right]\right| \rightarrow 0$ as $n \rightarrow \infty$ uniformly on $H$. Now $\left|\mathfrak{C}\left(X_{n}\right)-\mathfrak{C}(X)\right|=\frac{\left|\mathfrak{C}\left(\delta_{n}\right) \cdot \mathfrak{C}\left(X_{n}\right)-\mathfrak{C}\left(\delta_{n}\right) \cdot \mathfrak{C}(X)\right|}{\left|\mathfrak{C}\left(\delta_{n}\right)\right|}=\frac{\left|\mathfrak{C}\left(X_{n} *_{c} \delta_{n}\right)-\mathfrak{C}\left(X *_{c} \delta_{n}\right)\right|}{\left|\mathcal{C}\left(\delta_{n}\right)\right|}=$ $\frac{\left|\mathfrak{C}\left[\left(X_{n}-X\right) *_{c} \delta_{n}\right]\right|}{\left|\mathcal{C}\left(\delta_{n}\right)\right|} \leq \frac{\left|\mathfrak{C}\left[\left(X_{n}-X\right) *_{c} \delta_{n}\right]\right|}{r} \rightarrow 0$ as $n \rightarrow \infty$ uniformly on $H$.

Theorem 4.9. Let $g_{n}(s)=\int_{0}^{n}\left(1-\frac{t}{n}\right) F(t) \cos (t s) d t, \forall s \in[0, \infty), n \in \mathbb{N}$, where $F \in C([0, \infty))$ be arbitrary. Then, a necessary and sufficient condition for $F \in$ $\mathfrak{C}\left(\mathcal{B}_{c}^{1}\right)$ is that $g_{n} \in \mathcal{B}_{c}^{1}, \forall n \in \mathbb{N}$ and $\left(g_{n}\right)$ is $\delta$-convergent in $\mathcal{B}_{c}^{1}$.

Proof. Suppose there exists $X=\left[\frac{\left(h_{n}\right)}{\left(\eta_{n}\right)}\right] \in \mathcal{B}_{c}^{1}$ such that $F=\mathfrak{C}(X)$. Then

$$
\begin{aligned}
\left(g_{n}\right. & \left.*_{c} \eta_{m}\right)(u) \\
& =\frac{1}{2} \int_{0}^{\infty} g_{n}(s)\left[\eta_{m}(u+s)+\eta_{m}(|u-s|)\right] d s \\
& =\frac{1}{2} \int_{0}^{\infty}\left[\eta_{m}(u+s)+\eta_{m}(|u-s|)\right]\left(\int_{0}^{n}\left(1-\frac{t}{n}\right) \mathfrak{C}(X)(t) \cos (t s) d t\right) d s \\
& =\frac{1}{2} \int_{0}^{n}\left(1-\frac{t}{n}\right) \mathfrak{C}(X)(t) \int_{0}^{\infty}\left[\eta_{m}(u+s)+\eta_{m}(|u-s|)\right] \cos (t s) d s d t \\
& =\frac{1}{2} \int_{0}^{n}\left(1-\frac{t}{n}\right) \mathfrak{C}(X)(t) \int_{0}^{\infty} \eta_{m}(v)[\cos (t(v+u))+\cos (t(v-u))] d v d t \\
& =\int_{0}^{n}\left(1-\frac{t}{n}\right) \mathfrak{C}(X)(t) \int_{0}^{\infty} \eta_{m}(v) \cos (t v) \cos (t u) d v d t \\
& =\int_{0}^{n}\left(1-\frac{t}{n}\right) \mathfrak{C}(X)(t) \mathfrak{C}\left(\eta_{m}\right)(t) \cos (t u) d t \\
& =\int_{0}^{n}\left(1-\frac{t}{n}\right) \mathfrak{C}\left(h_{m}\right)(t) \cos (t u) d t, \text { by Lemma 4.4). }
\end{aligned}
$$

By using the proof of the necessity part of Theorem 2.11, we get $g_{n}{ }^{*}{ }_{c} \eta_{m} \in L^{1}([0, \infty))$ for $n, m \in \mathbb{N}$ and $g_{n} *_{c} \eta_{m} \rightarrow \pi h_{m}$ as $n \rightarrow \infty$ in $L^{1}([0, \infty))$, for each fixed $m \in \mathbb{N}$. If $X_{n}=\left[\frac{\left(g_{n} * \eta_{m}\right)}{\left(\eta_{m}\right)}\right], X=\left[\frac{\left(\pi h_{m}\right)}{\left(\eta_{m}\right)}\right]$, then necessity follows from Lemma 3.1.

To prove the sufficiency part, we assume that there exists $X=\left[\frac{\left(h_{m}\right)}{\left(\psi_{m}\right)}\right] \in \mathcal{B}_{c}^{1}$ such that $X_{n} \stackrel{\delta}{\rightarrow} X$ as $n \rightarrow \infty$ in $\mathcal{B}_{c}^{1}$ where $X_{n}=\left[\frac{\left(g_{n} *_{c} \psi_{k}\right)}{\left(\psi_{k}\right)}\right] \in \mathcal{B}_{c}^{1}$. This implies 
that $g_{n} *_{c} \psi_{m}=X_{n} *_{c} \psi_{m} \rightarrow X *_{c} \psi_{m}$ as $n \rightarrow \infty$ in $L^{1}([0, \infty))$, for each fixed $m \in \mathbb{N}$. By using similar arguments to those involved in the necessity part, we get $\left(g_{n} *_{c} \psi_{m}\right)=\mathcal{C}\left(f_{n, m}\right)$, where $f_{n, m}(t)=\chi_{[0, n]}(t)\left(1-\frac{t}{n}\right) F(t) \cdot \mathcal{C}\left(\psi_{m}\right)(t)$. Since $\mathcal{C}\left(f_{n, m}\right) \in L^{1}([0, \infty))$, we have $\mathcal{C}\left(g_{n} *_{c} \psi_{m}\right)=\mathcal{C}\left(\mathcal{C}\left(f_{n, m}\right)\right)=f_{n, m}$. Further, using the continuity of the Fourier cosine transform on $L^{1}([0, \infty))$, we also have $\mathfrak{C}\left(X *_{c} \psi_{m}\right)=$ $\lim _{n \rightarrow \infty} \mathcal{C}\left(g_{n} *_{c} \psi_{m}\right)=\lim _{n \rightarrow \infty} f_{n, m}=F \cdot \mathcal{C}\left(\psi_{m}\right)$. Since $\mathcal{C}\left(\psi_{m}\right) \rightarrow 1$ as $m \rightarrow \infty$, it follows that $\mathfrak{C}(X)=\lim _{m \rightarrow \infty} \mathcal{C}\left(h_{m}\right)=\lim _{m \rightarrow \infty} \mathfrak{C}\left(X *_{c} \psi_{m}\right)=\lim _{m \rightarrow \infty} F \cdot \mathcal{C}\left(\psi_{m}\right)=F$.

Definition 4.10. We define the extended Fourier sine transform $\mathfrak{S}(X)$ of $X=$ $\left[\left(f_{n}\right) /\left(\delta_{n}\right)\right] \in \mathcal{B}_{s}^{1}$ by $\mathfrak{S}(X)(t)=\lim _{n \rightarrow \infty} \mathcal{S}\left(f_{n}\right)(t), \quad \forall t \in[0, \infty)$.

Since the proofs of the following six theorems are analogous to those of the corresponding theorems for the extended Fourier cosine transform on $\mathcal{B}_{c}^{1}$, we leave out the details.

Lemma 4.11. (Consistency) For each $f \in L^{1}([0, \infty))$, we have $\mathfrak{S}(f)=\mathcal{S}(f)$.

Theorem 4.12. For $X=\left[\left(f_{n}\right) /\left(\delta_{n}\right)\right] \in \mathcal{B}_{s}^{1}, \mathfrak{C}\left(\delta_{k}\right) \cdot \mathfrak{S}(X)=\mathcal{S}\left(f_{k}\right), \forall k \in \mathbb{N}$.

Theorem 4.13. The extended Fourier sine transform $\mathfrak{S}: \mathcal{B}_{s}^{1} \rightarrow C([0, \infty))$ is linear.

Theorem 4.14. The extended Fourier sine transform $\mathfrak{S}: \mathcal{B}_{s}^{1} \rightarrow C([0, \infty))$ is one-to-one.

Theorem 4.15. The extended Fourier sine transform $\mathfrak{S}: \mathcal{B}_{s}^{1} \rightarrow C([0, \infty))$ is continuous with respect to $\delta$-convergence and $\Delta$-convergence.

Theorem 4.16. If $X \in \mathcal{B}_{s}^{1}$ and $h \in L^{1}([0, \infty))$, then $\mathfrak{S}\left(X *_{s c} h\right)=\mathfrak{S}(X) \cdot \mathcal{C}(h)$.

It is also interesting to note that if $X *_{s c} Y$ is defined by $\left[\left(f_{n} *_{s c} g_{n}\right) /\left(\phi_{n} *_{c} \psi_{n}\right)\right]$, for $X=\left[\left(f_{n}\right) /\left(\phi_{n}\right)\right] \in \mathcal{B}_{s}^{1}$ and $Y=\left[\frac{g_{n}}{\psi_{n}}\right] \in \mathcal{B}_{c}^{1}$, then $X *_{s c} Y \in \mathcal{B}_{s}^{1}$, and we have the following theorem.

Theorem 4.17.(Generalized convolution theorem) If $X \in \mathcal{B}_{s}^{1}$ and $Y \in \mathcal{B}_{c}^{1}$, then $\mathfrak{S}\left(X *_{s c} Y\right)=\mathfrak{S}(X) \cdot \mathcal{C}(Y)$.

Theorem 4.18. Let $F \in C([0, \infty))$. Then, $F \in \mathfrak{S}\left(\mathcal{B}_{s}^{1}\right)$ if and only if $\left(\varrho_{n}\right) \delta$ converges in $\mathcal{B}_{s}^{1}$, where $\varrho_{n}(s)=\int_{0}^{n}\left(1-\frac{t}{n}\right) F(t) \sin (t s) d t, \forall s \geq 0, \forall n \in \mathbb{N}$.

Proof. Suppose there exists $X=\left[\left(h_{n}\right) /\left(\eta_{n}\right)\right] \in \mathcal{B}_{s}^{1}$ such that $F=\mathfrak{S}(X)$. Then by Fubini's theorem and by a simple computation, we obtain that

$$
\begin{aligned}
& \left(\varrho_{n} *_{s c} \eta_{m}\right)(u) \\
& \quad=\frac{1}{2} \int_{0}^{n}\left(1-\frac{t}{n}\right) \mathfrak{S}(X)(t) \int_{0}^{\infty} \eta_{m}(v)[\sin (t(u+v))+\sin (t(u-v))] d v d t \\
& \quad=\int_{0}^{n}\left(1-\frac{t}{n}\right) \mathfrak{S}(X)(t) \int_{0}^{\infty} \eta_{m}(v) \sin (t u) \cos (t v) d v d t
\end{aligned}
$$




$$
\begin{aligned}
& =\int_{0}^{n}\left(1-\frac{t}{n}\right) \mathfrak{S}(X)(t) \cdot \mathcal{C}\left(\eta_{m}\right)(t) \sin (t u) d t \\
& =\int_{0}^{n}\left(1-\frac{t}{n}\right) \mathcal{S}\left(h_{m}\right)(t) \sin (t u) d t \text { (by Theorem 4.12). }
\end{aligned}
$$

From the proof of Theorem 2.18, we get $\varrho_{n} *_{s c} \eta_{m} \in L^{1}([0, \infty)), \forall n, m \in \mathbb{N}$ and for each $m \in \mathbb{N}, \varrho_{n} *_{s c} \eta_{m} \rightarrow \pi h_{m}$ as $n \rightarrow \infty$ in $L^{1}([0, \infty))$. If $X_{n}=\left[\left(\varrho_{n} *_{s c} \eta_{m}\right) /\left(\eta_{m}\right)\right]$, $X=\left[\left(\pi h_{m}\right) /\left(\eta_{m}\right)\right]$, then necessity follows by using the fact that $X_{n} *_{s c} \eta_{m}=$ $\varrho_{n} *_{s c} \eta_{m}$ and $X *_{s c} \eta_{m}=\pi h_{m}, \forall n, m \in \mathbb{N}$.

Suppose that there exists $X=\left[\left(h_{m}\right) /\left(\psi_{m}\right)\right] \in \mathcal{B}_{s}^{1}$ such that $X_{n} \stackrel{\delta}{\rightarrow} X$ as $n \rightarrow \infty$ in $\mathcal{B}_{s}^{1}$, where $X_{n}=\left[\left(\varrho_{n} *_{s c} \psi_{k}\right) /\left(\psi_{k}\right)\right] \in \mathcal{B}_{s}^{1}$. Then, for each $m \in \mathbb{N}, \varrho_{n} *_{s c} \psi_{m}=$ $X_{n} *_{s c} \psi_{m} \rightarrow X *_{s c} \psi_{m}$ as $n \rightarrow \infty$ in $L^{1}([0, \infty))$. Arguing as before, we have $\left(\varrho_{n} *_{s c}\right.$ $\left.\psi_{m}\right)=\mathcal{S}\left(f_{n, m}\right)$, where $f_{n, m}(t)=\chi_{[0, n]}(t)\left(1-\frac{t}{n}\right) F(t) \cdot \mathcal{C}\left(\psi_{m}\right)(t), \forall t \geq 0$. Since $\mathcal{S}\left(f_{n, m}\right) \in L^{1}([0, \infty))$, we have $\mathcal{S}\left(\varrho_{n} *_{s c} \psi_{m}\right)=\mathcal{S}\left(\mathcal{S}\left(f_{n, m}\right)\right)=f_{n, m}$. Further, we have $\mathcal{S}\left(X *_{s c} \psi_{m}\right)=\lim _{n \rightarrow \infty} \mathcal{S}\left(\varrho_{n} *_{s c} \psi_{m}\right)=\lim _{n \rightarrow \infty} f_{n, m}=F \cdot \mathcal{C}\left(\psi_{m}\right)$. Now by using Lemma 4.1 , we get $\mathfrak{S}(X)=\lim _{m \rightarrow \infty} \mathfrak{S}\left(h_{m}\right)=\lim _{m \rightarrow \infty} \mathcal{S}\left(X *_{s c} \psi_{m}\right)=\lim _{m \rightarrow \infty} F \cdot \mathcal{C}\left(\psi_{m}\right)=F$.

Acknowledgement. The authors wish to express their sincere thanks to the anonymous Referee for valuable comments which helped to substantially improve the presentation of this paper.

\section{References}

[1] T. K. Boehme, The support of Mikusinski operators, Trans. Amer. Math. Soc., 176(1973), 319-334.

[2] V. A. Kakichev, N. X Thao and V. K. Tuan, On the generalized convolutions for Fourier cosine and sine transforms, East-West J. Math., 1(1998), 85-90.

[3] Y. Katznelson, An introduction to harmonic analysis, Dover, (1976).

[4] P. J. Miana, Convolutions, Fourier trigonometric transforms and applications, Integral Transform. Spec. Funct., 16(2005), 583-585.

[5] J. Mikusiński and P. Mikusiński, Quotients de suites et leurs applications dans l'analyse fonctionnelle, C.R. Acad. Sc. Paris, 293, Serie I (1981), 463-464.

[6] P. Mikusiński, Convergence of Boehmians, Japan. J. Math. (N.S.), 9(1983), 159-179.

[7] P. Mikusiński, The Fourier transform of tempered Boehmians, Fourier Analysis, Lecture Notes in Pure and Appl. Math., Marcel Dekker, New York, (1994), 303-309.

[8] P. Mikusiński, Tempered Boehmians and ultra distributions, Proc. Amer. Math. Soc., 123(1995), 813-817.

[9] P. Mikusiński, On flexibility of Boehmians, Integral transform. Spec. Funct., 4(1996), 141-146.

[10] W. Rudin, Real and complex analysis, McGraw-Hill Book Company, New York, 1987. 
[11] I. N. Sneddon, The use of integral transforms, McGraw-Hill International Editions, New York, 1972.

[12] E. C. Titchmarsh, Introduction to the theory of Fourier integrals, Oxford University Press, 1948. 